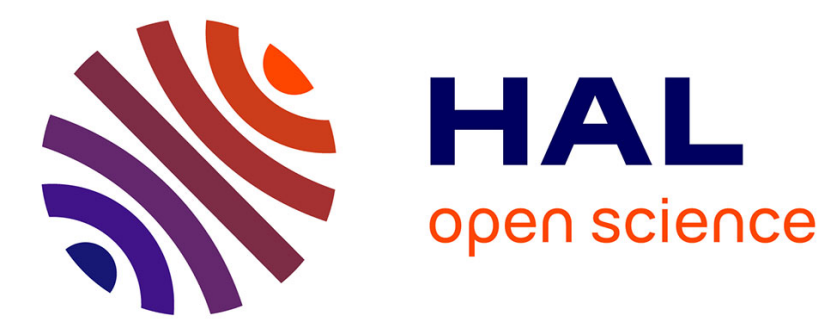

\title{
FREE DIVISORS IN A PENCIL OF CURVES
}

Jean Vallès

\section{To cite this version:}

Jean Vallès. FREE DIVISORS IN A PENCIL OF CURVES. Journal of Singularities, 2016, 14, pp.1-2. 10.5427/jsing.2016.14a . hal-01114265v2

\section{HAL Id: hal-01114265 \\ https://hal.science/hal-01114265v2}

Submitted on 12 Jan 2016

HAL is a multi-disciplinary open access archive for the deposit and dissemination of scientific research documents, whether they are published or not. The documents may come from teaching and research institutions in France or abroad, or from public or private research centers.
L'archive ouverte pluridisciplinaire HAL, est destinée au dépôt et à la diffusion de documents scientifiques de niveau recherche, publiés ou non, émanant des établissements d'enseignement et de recherche français ou étrangers, des laboratoires publics ou privés. 


\title{
FREE DIVISORS IN A PENCIL OF CURVES
}

\author{
JEAN VALLÈS
}

\begin{abstract}
A plane curve $D \subset \mathbb{P}^{2}(\boldsymbol{k})$ where $\boldsymbol{k}$ is a field of characteristic zero is free if its associated sheaf $\mathcal{T}_{D}$ of vector fields tangent to $D$ is a free $\mathscr{O}_{\mathbb{P}^{2}(\boldsymbol{k})}$-module (see [7] or [5] for a definition in a more general context). Relatively few free curves are known. Here we prove that a divisor $D$ consisting of a union of curves of a pencil of plane projective curves with the same degree and with a smooth base locus is a free divisor if and only if $D$ contains all the singular members of the pencil and its Jacobian ideal is locally a complete intersection.
\end{abstract}

\section{INTRODUCTION}

Let $\boldsymbol{k}$ be a field of characteristic zero and let $S=\boldsymbol{k}[x, y, z]$ be the graded ring such that $\mathbb{P}^{2}=\operatorname{Proj}(S)$. We write $\partial_{x}:=\frac{\partial}{\partial x}, \partial_{y}:=\frac{\partial}{\partial y}, \partial_{z}:=\frac{\partial}{\partial z}$ and $\nabla F=\left(\partial_{x} F, \partial_{y} F, \partial_{z} F\right)$ for a homogenous polynomial $F \in S$.

Let $D=\{F=0\}$ be a reduced curve of degree $n$. The kernel $\mathcal{T}_{D}$ of the map $\nabla F$ is a rank two reflexive sheaf, hence a vector bundle on $\mathbb{P}^{2}$. It is the rank two vector bundle of vector fields tangent along $D$, defined by the following exact sequence:

$$
0 \longrightarrow \mathcal{T}_{D} \longrightarrow \mathscr{O}_{\mathbb{P}^{2}}^{3} \stackrel{\nabla F}{\longrightarrow} \mathcal{J}_{\nabla F}(n-1) \longrightarrow 0,
$$

where the sheaf $\mathcal{J}_{\nabla F}$ (also denoted $\mathcal{J}_{\nabla D}$ in this text) is the Jacobian ideal of $F$. Set theoretically $\mathcal{J}_{\nabla F}$ defines the singular points of the divisor $D$. For instance if $D$ consists of $s$ generic lines then $\mathcal{J}_{\nabla D}$ defines the set of $\left(\begin{array}{l}s \\ 2\end{array}\right)$ vertices of $D$.

Remark 1.1. A non zero section $s \in \mathrm{H}^{0}\left(\mathcal{T}_{D}(a)\right)$, for some shift $a \in \mathbb{N}$, corresponds to a derivation $\delta=P_{a} \partial_{x}+Q_{a} \partial_{y}+R_{a} \partial_{z}$ verifying $\delta(F)=0$, where $\left(P_{a}, Q_{a}, R_{a}\right) \in \mathrm{H}^{0}\left(\mathscr{O}_{\mathbb{P}^{2}}(a)\right)^{3}$.

In some particular cases that can be found in [5], $\mathcal{T}_{D}$ is a free $\mathscr{O}_{\mathbb{P}^{2}}$-module; it means that there are two vector fields of degrees $a$ and $b$ that form a basis of $\bigoplus_{n} \mathrm{H}^{0}\left(\mathcal{T}_{D}(n)\right)(D$ is said to be free with exponents $(a, b))$; it arises, for instance, when $D$ is the union of the nine inflection lines of a smooth cubic curve. The notion of free divisor was introduced by Saito [7] for reduced divisors and studied by Terao [11] for hyperplane arrangements. Here we recall a definition of freeness for projective curves. For a more general definition we refer to Saito [7].

Definition 1.2. A reduced curve $D \subset \mathbb{P}^{2}$ is free with exponents $(a, b) \in \mathbb{N}^{2}$ if

$$
\mathcal{T}_{D} \simeq \mathscr{O}_{\mathbb{P}^{2}}(-a) \oplus \mathscr{O}_{\mathbb{P}^{2}}(-b) .
$$

A smooth curve of degree $\geq 2$ is not free, an irreducible curve of degree $\geq 3$ with only nodes and cusps as singularities is not free (see [1, Example 4.5]). Actually few examples

2010 Mathematics Subject Classification. 14C21, 14N20, 32S22, $14 \mathrm{H} 50$.

Key words and phrases. Arrangements of curves, Pencil of curves, Freeness of arrangements, Logarithmic sheaves.

Author partially supported by ANR GEOLMI ANR-11-BS03-0011 and PHC-SAKURA 31944VE. 
of free curves are known and of course very few families of free curves are known. One such family can be found in [10, Prop. 2.2].

In a personal communication it was conjectured by E. Artal and J.I. Cogolludo that the union of all the singular members of a pencil of plane curves (assuming that the general one is smooth) should be free. Three different cases occur:

- the base locus is smooth (for instance the union of six lines in a pencil of conics passing through four distinct points);

- the base locus is not smooth but every curve in the pencil is reduced (for instance the four lines in a pencil of conics where two of the base points are infinitely near points);

- the base locus is not smooth and there exists exactly one non reduced curve in the pencil (for instance three lines in a pencil of bitangent conics).

In the third case the divisor of singular members is not reduced but its reduced structure is expected to be free.

We point out that if two distinct curves of the pencil are not reduced then all curves are singular. Even in this case, we believe that a free divisor can be obtained by chosing a finite number of reduced components through all the singular points.

In this paper we prove (see theorem 2.7) that a divisor $D$ consisting of a union of curves of a pencil of degree $n$ plane curves with a smooth base locus (i.e. the base locus consists of $n^{2}$ distinct points) is a free divisor with determined exponents if and only if $D$ contains all the singular members of the pencil and its Jacobian ideal is locally a complete intersection (this is always the case when $D$ is a union of lines or when the singular points of $D$ are all ordinary double points). More generally, we describe the vector bundle of logarithmic vector fields tangent to any union of curves of the pencil (see theorem 2.10) by studying one particular vector field "canonically tangent" to the pencil, that is introduced in the key lemma 2.1.

This gives already a new and easy method to produce free divisors.

I thank J. I. Cogolludo for his useful comments.

\section{Pencil of Plane CuRves}

2.1. Generalities and notations. Let $\{f=0\}$ and $\{g=0\}$ be two reduced curves of degree $n \geq 1$ with no common component. For any $(\alpha, \beta) \in \mathbb{P}^{1}$ the curve $C_{\alpha, \beta}$ is defined by the equation $\{\alpha f+\beta g=0\}$ and $\mathcal{C}(f, g)=\left\{C_{\alpha, \beta} \mid(\alpha, \beta) \in \mathbb{P}^{1}\right\}$ is the pencil of all these curves.

In section 2 we will assume that the general member of the pencil $\mathcal{C}(f, g)$ is a smooth curve and that $C_{\alpha, \beta}$ is reduced for every $(\alpha, \beta) \in \mathbb{P}^{1}$.

Under these assumptions there are finitely many singular curves in $\mathcal{C}(f, g)$ but also finitely many singular points. We recall that the degree of the discriminant variety of degree $n$ curves is $3(n-1)^{2}$ (it is a particular case of the Boole formula; see [12, Example 6.4]). Since the general curve in the pencil is smooth, the line defined by the pencil $\mathcal{C}(f, g)$ in the space of degree $n$ curves meets the discriminant variety along a finite scheme of length $3(n-1)^{2}$ (not empty for $n \geq 2$ ). The number of singular points is of course related to the multiplicity of the singular curves in the pencil as we will see below.

Let us fix some notation. The scheme defined by the ideal sheaf $\mathcal{J}_{\nabla C_{\alpha_{i}, \beta_{i}}}$ is denoted by $Z_{\alpha_{i}, \beta_{i}}$. The union of all the singular members of the pencil $\mathcal{C}(f, g)$ form a divisor $D^{\mathrm{sg}}$. A 
union of $k \geq 2$ distinct members of $\mathcal{C}(f, g)$ is denoted by $D_{k}$. The whole set of singularities of the pencil is denoted by $\operatorname{Sing}(\mathcal{C})$.

2.2. Derivation tangent to a smooth pencil. Let us consider the following derivation, associated "canonically" to the pencil:

Lemma 2.1. For any union $D_{k}$ of $k \geq 1$ members of the pencil there exists a non zero section $s_{\delta, k} \in \mathrm{H}^{0}\left(\mathcal{T}_{D_{k}}(2 n-2)\right)$ induced by the derivation

$\delta=(\nabla f \wedge \nabla g) \cdot \nabla=\left(\partial_{y} f \partial_{z} g-\partial_{z} f \partial_{y} g\right) \partial_{x}+\left(\partial_{z} f \partial_{x} g-\partial_{x} f \partial_{z} g\right) \partial_{y}+\left(\partial_{x} f \partial_{y} g-\partial_{y} f \partial_{x} g\right) \partial_{z}$.

Proof. Since $\delta(\alpha f+\beta g)=\operatorname{det}(\nabla f, \nabla g, \nabla(\alpha f+\beta g))=0$ we have for any $k \geq 1$,

$$
\delta(f)=\delta(g)=\delta(\alpha f+\beta g)=\delta\left(\prod_{i=1}^{k}\left(\alpha_{i} f+\beta_{i} g\right)\right)=0 .
$$

According to the remark 1.1] it gives the desired section.

Let us introduce a rank two sheaf $\mathcal{F}$ defined by the following exact sequence:

$$
0 \longrightarrow \mathscr{O}_{\mathbb{P}^{2}}(2-2 n) \stackrel{\nabla f \wedge \nabla g}{\longrightarrow} \mathscr{O}_{\mathbb{P}^{2}}^{3} \longrightarrow \mathcal{F} \longrightarrow 0 .
$$

If we denote by $\operatorname{sg}(\mathcal{F})$ the singular scheme of $\mathcal{F}$ supported by the set $\left\{p \in \mathbb{P}^{2} \mid \operatorname{rank}(\mathcal{F} \otimes\right.$ $\left.\left.\mathscr{O}_{p}\right)>2\right\}$ of singular points of $\mathcal{F}$, we have:

Lemma 2.2. A point $p \in \mathbb{P}^{2}$ belongs to $\operatorname{sg}(\mathcal{F})$ if and only if two smooth members of the pencil share the same tangent line at $p$ or one curve of the pencil is singular at $p$. Moreover $\operatorname{sg}(\mathcal{F})$ is a finite closed scheme with length $l(\operatorname{sg}(\mathcal{F}))=3(n-1)^{2}$.

Remark 2.3. Let us precise that if two smooth members intersect then all the smooth members of the pencil intersect with the same tangency.

Remark 2.4. If the base locus of $\mathcal{C}(f, g)$ consists of $n^{2}$ distinct points then two curves of the pencil meet transversaly at the base points and $p \in \operatorname{sg}(\mathcal{F})$ if and only if $p$ is a singular point for a unique curve $C_{\alpha, \beta}$ in the pencil and does not belong to the base locus. One can assume that $p \in \operatorname{sg}(\mathcal{F})$ is singular for $\{f=0\}$. In other words, when the base locus is smooth, the support of $\operatorname{sg}(\mathcal{F})$ is $\operatorname{Sing}(\mathcal{C})$.

Proof. The support of the $\operatorname{sg}(\mathcal{F})$ is also defined by $\left\{p \in \mathbb{P}^{2} \mid(\nabla f \wedge \nabla g)(p)=0\right\}$. The zero scheme defined by $\nabla f \wedge \nabla g$ and $\nabla(\alpha f+\beta g) \wedge \nabla g$ are clearly the same; it means that the singular points of any member in the pencil is a singular point for $\mathcal{F}$. One can also obtain $(\nabla f \wedge \nabla g)(p)=0$ at a smooth point when the vectors $(\nabla f)(p)$ and $(\nabla g)(p)$ are proportional i.e. when two smooth curves of the pencil share the same tangent line at $p$.

Since every curve of the pencil is reduced $\operatorname{sg}(\mathcal{F})$ is finite, its length can be computed by writing the resolution of the ideal $\mathcal{J}_{\operatorname{sg}(\mathcal{F})}$ (for a sheaf of ideal $\mathcal{J}_{Z}$ defining a finite scheme $Z$ of length $l(Z)$, we have $\left.c_{2}\left(\mathcal{J}_{Z}\right)=l(Z)\right)$. Indeed, if we dualize the following exact sequence

$$
0 \longrightarrow \mathscr{O}_{\mathbb{P}^{2}}(2-2 n) \stackrel{\nabla f \wedge \nabla g}{\longrightarrow} \mathscr{O}_{\mathbb{P}^{2}}^{3} \longrightarrow \mathcal{F} \longrightarrow 0
$$

we find, according to Hilbert-Burch theorem,

$$
0 \longrightarrow \mathcal{F}^{\vee} \stackrel{(\nabla f, \nabla g)}{\longrightarrow} \mathscr{O}_{\mathbb{P}^{2}}^{3} \stackrel{\nabla f \wedge \nabla g}{\longrightarrow} \mathscr{O}_{\mathbb{P}^{2}}(2 n-2) .
$$

It proves that $\mathcal{F}^{\vee}=\mathscr{O}_{\mathbb{P}^{2}}(1-n)^{2}$ and that the image of the last map is $\mathcal{J}_{\mathrm{sg}(\mathcal{F})}(2 n-2)$.

Then $l(\operatorname{sg}(\mathcal{F}))=3(n-1)^{2}$. We point out that this number is the degree of the discriminant variety of degree $n$ curves. 
Now let us call $D_{k}$ the divisor defined by $k \geq 2$ members of the pencil and let us consider the section $s_{\delta, k} \in \mathrm{H}^{0}\left(\mathcal{T}_{D_{k}}(2 n-2)\right)$ corresponding (see remark 1.1) to the derivation $\delta$. Let $Z_{k}:=Z\left(s_{\delta, k}\right)$ be the zero locus of $s_{\delta, k}$.

Lemma 2.5. The section $s_{\delta, k}$ vanishes in codimension at least two.

Proof. Let us consider the following commutative diagram

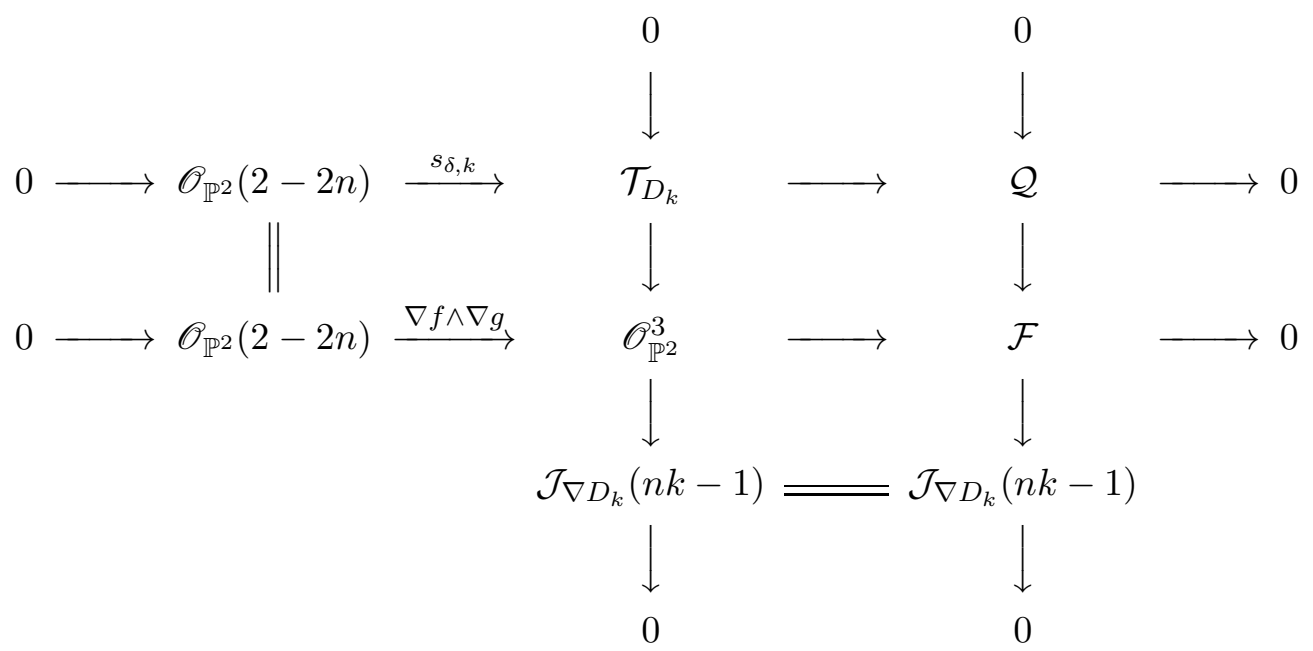

where $\mathcal{Q}=\operatorname{coker}\left(s_{\delta, k}\right)$. Assume that $Z_{k}$ contains a divisor $H$. Tensor now the last vertical exact sequence of the above diagram by $\mathscr{O}_{p}$ for a general point $p \in H$. Since $p$ does not belong to the Jacobian scheme defined by $\mathcal{J}_{\nabla D_{k}}$ we have $\mathcal{J}_{\nabla D_{k}} \otimes \mathscr{O}_{p}=\mathscr{O}_{p}$ and $\operatorname{Tor}_{1}\left(\mathcal{J}_{\nabla D_{k}}, \mathscr{O}_{p}\right)=0$. Since $p \in H \subset Z_{k}$ we have $\operatorname{rank}\left(\mathcal{Q} \otimes \mathscr{O}_{p}\right) \geq 2$; it implies $\operatorname{rank}\left(\mathcal{F} \otimes \mathscr{O}_{p}\right) \geq 3$ in other words that $p \in \operatorname{sg}(\mathcal{F})$; this contradicts $\operatorname{codim}\left(\operatorname{sg}(\mathcal{F}), \mathbb{P}^{2}\right) \geq 2$, proved in lemma 2.2

Then $\mathcal{Q}$ is the ideal sheaf of the codimension two scheme $Z_{k}$, i.e. $\mathcal{Q}=\mathcal{J}_{Z_{k}}(n(2-k)-1)$ and we have an exact sequence

$$
0 \longrightarrow \mathcal{J}_{Z_{k}}(n(2-k)-1) \longrightarrow \mathcal{F} \longrightarrow \mathcal{J}_{\nabla D_{k}}(n k-1) \longrightarrow 0 .
$$

From this commutative diagram we obtain the following lemma.

Lemma 2.6. Let $D_{k}$ be a union of $k \geq 2$ members of $\mathcal{C}(f, g)$. Then

$$
c_{2}\left(\mathcal{J}_{\nabla D_{k}}\right)+c_{2}\left(\mathcal{J}_{Z_{k}}\right)=3(n-1)^{2}+n^{2}(k-1)^{2} .
$$

Proof. According to the above commutative diagram we compute $c_{2}(\mathcal{F})$ in two different ways. The horizontal exact sequence gives $c_{2}(\mathcal{F})=4(n-1)^{2}$ when the vertical one gives $c_{2}(\mathcal{F})=c_{2}\left(\mathcal{J}_{\nabla D_{k}}\right)+c_{2}\left(\mathcal{J}_{Z_{k}}\right)+(n-1)^{2}-n^{2}(k-1)^{2}$. The lemma is proved by eliminating $c_{2}(\mathcal{F})$.

2.3. Free divisors in the pencil. When $D_{k}$ contains the divisor $D^{\mathrm{sg}}$ of all the singular members of the pencil we show now that, under some supplementary condition on the nature of the singularities, it is free with exponents $(2 n-2, n(k-2)+1)$.

Theorem 2.7. Assume that the base locus of the pencil $\mathcal{C}(f, g)$ is smooth, $n \geq 1$ and $k>1$. Then, $D_{k}$ is free with exponents $(2 n-2, n(k-2)+1)$ if and only if $D_{k} \supseteq D^{\mathrm{sg}}$ and $J_{\nabla D_{k}}$ is locally a complete intersection at every $p \in \operatorname{Sing}(\mathcal{C})$. 
Proof. Let us remark first that $D_{k}$ is free with exponents $(2 n-2, n(k-2)+1)$ if and only if the zero set $Z_{k}$ of the "canonical section" $s_{\delta, k}$ is empty. Indeed, if $Z_{k}=\emptyset$ then $\mathrm{H}^{1}\left(\mathcal{T}_{D_{k}}(m)\right)=0$ for all $m \in \mathbb{Z}$ and by Horrocks'criterion it implies that $D_{k}$ is free with exponents $(2 n-2, n(k-2)+1)$. The other direction is straitforward.

According to lemma 2.6, $Z_{k}=\emptyset$ if and only if $c_{2}\left(\mathcal{J}_{\nabla D_{k}}\right)=n^{2}(k-1)^{2}+3(n-1)^{2}$. Moreover it is well known (see [9, section 1.3] for instance) that the length of the Jacobian scheme of $D_{k}$ is $c_{2}\left(\mathcal{J}_{\nabla D_{k}}\right)=\sum_{p \in \operatorname{Sing}\left(D_{k}\right)} \tau_{p}\left(D_{k}\right)$ where $\tau_{p}\left(D_{k}\right)$ is the Tjurina number of $D_{k}$ at $p \in D_{k}$ (this number $\tau_{p}\left(D_{k}\right)$ is the length of the subscheme of the Jacobian scheme supported by $p$ ). Then to prove the theorem we show below that $\sum_{p \in \operatorname{Sing}\left(D_{k}\right)} \tau_{p}\left(D_{k}\right)=n^{2}(k-1)^{2}+3(n-1)^{2}$ if and only if $D_{k} \supseteq D^{\mathrm{sg}}$ and $J_{\nabla D_{k}}$ is locally a complete intersection at every $p \in \operatorname{Sing}(\mathcal{C})$.

The Jacobian scheme of $D_{k}$ is supported by the base locus $B$ of the pencil and by the singular points of the $k$ curves forming $D_{k}$. The syzygy $\nabla f \wedge \nabla g$ of $J_{\nabla D_{k}}$ does not vanish at $p \in B$; it implies that $J_{\nabla D_{k}}$ is locally a complete intersection at $p \in B$; according to [9, section 1.3] it gives $\tau_{p}\left(D_{k}\right)=\mu_{p}\left(D_{k}\right)$, where this last number is the Milnor number of $D_{k}$ at $p$. Since $p$ is an ordinary singular point of multiplicity $k$ we obtain $\mu_{p}\left(D_{k}\right)=(k-1)^{2}$. Then $\sum_{p \in B} \tau_{p}\left(D_{k}\right)=n^{2}(k-1)^{2}$.

Let us compute now $\sum_{p \in \operatorname{Sing}\left(D_{k}\right) \backslash B} \tau_{p}\left(D_{k}\right)$.

Let $C_{p} \subset D_{k}$ be the unique curve in the pencil singular at $p \in \operatorname{Sing}\left(D_{k}\right) \backslash B$. We can verify without difficulties that their Jacobian ideals coincide locally at $p \in \operatorname{Sing}\left(D_{k}\right) \backslash B$, in particular $\tau_{p}\left(D_{k}\right)=\tau_{p}\left(C_{p}\right)$ and $\sum_{p \in \operatorname{Sing}\left(D_{k}\right) \backslash B} \tau_{p}\left(D_{k}\right)=\sum_{p \in \operatorname{Sing}\left(D_{k}\right) \backslash B} \tau_{p}\left(C_{p}\right)$.

Let $I=(\nabla f \wedge \nabla g)$ be the ideal generated by the two by two minors of the $3 \times 2$ matrix $(\nabla f, \nabla g)$ defining the scheme $\operatorname{sg}(\mathcal{F})$. Let $\operatorname{sg}(\mathcal{F})_{p}$ be the subscheme of $\operatorname{sg}(\mathcal{F})$ supported by the point $p$. We have seen in lemma 2.2 that $\operatorname{sg}(\mathcal{F})$ is supported by the whole set of singular points of the pencil and that $l(\operatorname{sg}(\mathcal{F}))=\sum_{p \in \operatorname{Sing}(\mathcal{C})} l\left(\operatorname{sg}(\mathcal{F})_{p}\right)=3(n-1)^{2}$. Let us consider the situation in a fixed point $p \in \operatorname{Sing}\left(D_{k}\right) \backslash B$. To simplify the notation assume that $f=0$ is an equation for $C_{p}$. Then the other curves of the pencil do not pass through $p$, in particular $g(p) \neq 0$. Since $<\nabla f \wedge \nabla g, \nabla g>=0, \nabla g$ is a syzygy of $I$ that do not vanish at $p \in \operatorname{Sing}\left(D_{k}\right) \backslash B$. It implies that $I$ is locally a complete intersection at $p$. Since the ideal $I_{p}$ is obtained by taking the two by two minors of the matrix $(\nabla f, \nabla g)$ in the local ring $S_{p}$ the inclusion $I_{p} \subset J_{\nabla f, p}$ is straitforward; This inclusion implies $\tau_{p}\left(C_{p}\right) \leq l\left(\operatorname{sg}(\mathcal{F})_{p}\right)$ because $l\left(\operatorname{sg}(\mathcal{F})_{p}\right)=l\left(S_{p} / I_{p}\right)$.

Then $Z_{k}=\emptyset$ if and only if $\sum_{p \in \operatorname{Sing}\left(D_{k}\right) \backslash B} \tau_{p}\left(C_{p}\right)=\sum_{p \in \operatorname{Sing}(\mathcal{C})} l\left(\operatorname{sg}(\mathcal{F})_{p}\right)$. And this equality is verified if and only if $\operatorname{Sing}\left(D_{k}\right) \backslash B=\operatorname{Sing}(\mathcal{C})$ and $l\left(\operatorname{sg}(\mathcal{F})_{p}\right)=\tau_{p}\left(C_{p}\right)$ for all $p \in \operatorname{Sing}(\mathcal{C})$. The second equality is equivalent to the equality $I_{p}=J_{\nabla f, p}$ which implies that the Jacobian ideal of $C_{p}$ is locally a complete intersection at $p$.

Since the Jacobian ideals of $D_{k}$ and $C_{p}$ coincide locally at $p \in \operatorname{Sing}\left(D_{k}\right) \backslash B$ this proves that $Z_{k}=\emptyset$ if and only if $D_{k}$ contains all the singular members of the pencil ( $\left.\operatorname{Sing}\left(D_{k}\right) \backslash B=\operatorname{Sing}(\mathcal{C})\right)$ and the Jacobian ideal of $D_{k}$ is locally a complete intersection in every singular point of the pencil $\left(\mu_{p}\left(C_{p}\right)=\tau_{p}\left(C_{p}\right)\right.$ for all $\left.p \in \operatorname{Sing}(\mathcal{C})\right)$.

Remark 2.8. We insist on the following fact: if the Jacobian ideal of $D_{k}$ is not locally a complete intersection at $p$ then $Z_{k}$ is not empty because $p \in \operatorname{supp}\left(Z_{k}\right)$ even if $D_{k}$ contains all the singular curves.

Remark 2.9. Except for $k=1$ and $(k, n)=(3, n)$ with $n \geq 5$ we can omit to precise the splitting type of $\mathcal{T}_{D_{k}}$. Moreprecisely, when $k \neq 1$ and $(k, n) \neq(3, n)$ with $n \geq 5$ the following equivalence is true: 
The divisor $D_{k}$ is free if and only if $D_{k} \supseteq D^{\mathrm{sg}}$ and the Jacobian ideal of $D_{k}$ is locally a complete intersection.

Let us prove it.

- It is not true for $k=1$. Indeed, one can choose a free curve $\{f=0\}$ and take any other singular curve $\{g=0\}$ with the same degree such that the intersection locus $\{f=g=0\}$ is smooth. Then $\{f=0\}$ is free even if it does not pass through all the singular points of the pencil.

- When $k=2$ it is true. Under the hypothesis of smoothness of the base locus of the pencil, there is only one example of union of $k=2$ curves that is free: the union of two lines (i.e. $k=2$ and $n=1$ ). Indeed, assume that $k=2$ and that $D_{2}$ is free. Then we have for $0 \leq t \leq n-1$ :

$$
0 \longrightarrow \mathscr{O}_{\mathbb{P}^{2}}(2-2 n) \stackrel{s_{\delta, 2}}{\longrightarrow} \mathscr{O}_{\mathbb{P}^{2}}(2-2 n+t) \oplus \mathscr{O}_{\mathbb{P}^{2}}(-1-t) \longrightarrow \mathcal{J}_{Z_{2}}(-1) \longrightarrow 0 .
$$

The Jacobian ideal of $D_{2}$ defines a finite scheme of length smaller or equal to $2(n-1)^{2}+n^{2}$ since for one reduced curve of degree $n$ the maximal length of its Jacobian scheme is $(n-1)^{2}$, attained for $n$ concurrent lines. Then, according to lemma 2.6, the length of the zero scheme $Z_{2}$ is at least $(n-1)^{2}$. When $n=1$ we have $\mathcal{T}_{D_{2}}=\mathscr{O}_{\mathbb{P}^{2}} \oplus \mathscr{O}_{\mathbb{P}^{2}}(-1)$ and $Z_{2}=\emptyset$. Otherwise, when $n>1, Z_{2}$ cannot be empty. This implies that $t>0$ when $n>1$. The zero scheme $Z_{2}$ is then a complete intersection $(t, 2 n-3-t)$; its length is $t(2 n-3-t)$ and this number should be greater than $(n-1)^{2}$. This never happens for $n>1$. We have shown that the union of two curves of degree $n>1$ meeting along $n^{2}$ distinct points is never free. If we accept a non smooth base locus then it is possible. Consider for instance an arrangement of four lines with a triple point. This arrangement is free with exponents $(1,2)$ and it can be seen as the union of the (only) two singular conics of a pencil of tangent conics (at the triple point).

- When $k=3$ it is true for $n<5$ but it is still an open question for $n \geq 5$. Indeed we have for $k=3$

$$
0 \longrightarrow \mathscr{O}_{\mathbb{P}^{2}} \stackrel{s_{\delta, 3}}{\longrightarrow} \mathcal{T}_{D_{3}}(2 n-2) \longrightarrow \mathcal{J}_{Z_{3}}(n-3) \longrightarrow 0 .
$$

If $n \leq 4, D_{3}$ is free and there is only one possible splitting for $\mathcal{T}_{D_{3}}(4)$, that is $\mathscr{O}_{\mathbb{P}^{2}} \oplus \mathscr{O}_{\mathbb{P}^{2}}(-1)$ for $n=2, \mathscr{O}_{\mathbb{P}^{2}}^{2}$ for $n=3$ and $\mathscr{O}_{\mathbb{P}^{2}} \oplus \mathscr{O}_{\mathbb{P}^{2}}(1)$ for $n=4$. It implies necessarly that $Z_{3}=\emptyset$. Then the Jacobian scheme of the union of the three cubics has exactly length $12+9 \times 4$ (resp. $27+16 \times 4$ ). In other words the sum of the Tjurina numbers of each cubic (resp. quartic) is 4 (resp. is 9). This is possible if and only if each cubic (resp. quartic) is a product of three (resp. four) concurrent lines. The pencil is equivalent to $\left(x^{3}-y^{3}, y^{3}-z^{3}\right)$ (resp. $\left(x^{4}-y^{4}, y^{4}-z^{4}\right)$ ) and it contains also the cubic form $x^{3}-z^{3}$ (resp. $\left.x^{4}-z^{4}\right)$.

More generally, if $k=3$ and $Z_{3}=\emptyset$ then $\mathrm{H}^{1}\left(\mathcal{T}_{D_{3}}(m)\right)=0$ for all $m \in \mathbb{Z}$ and by Horrocks' criterion it implies $\mathcal{T}_{D_{3}}(2 n-2)=\mathscr{O}_{\mathbb{P}^{2}} \oplus \mathscr{O}_{\mathbb{P}^{2}}(n-3)$. This arises if and only if the pencil is projectively equivalent to $\left(x^{n}-y^{n}, y^{n}-z^{n}\right)$.

But if $n \geq 5$ one could possibly have $D_{3}$ free but $Z_{3} \neq \emptyset$; i.e. $\mathcal{T}_{D_{3}}(2 n-2)=\mathscr{O}_{\mathbb{P}^{2}}(t) \oplus$ $\mathscr{O}_{\mathbb{P}^{2}}(n-3-t)$ with $1 \leq t \leq \frac{n-3}{2}$ and $Z_{3}$ is a non empty complete intersection $t(n-3-t)$. In other words $Z_{3}$ is supported by the singularities where $D_{3}$ is not a local complete intersection and by the singularities that do not belong $D_{3}$.

- When $k \geq 4$ it is true. The inequality $k \geq 4$ gives $n(k-2)+1>2 n-2$ for all $n$ and there is only one possible splitting for $\mathcal{T}_{D_{k}}$ that is $\mathscr{O}_{\mathbb{P}^{2}}(2-2 n) \oplus \mathscr{O}_{\mathbb{P}^{2}}(n(2-k)-1)$. Then $D_{k}$ is free if and only if $Z_{k}=\emptyset$. 
2.4. Singular members ommitted. When $D_{k} \supset D^{\mathrm{sg}}$ and its Jacobian is locally a complete intersection we have seen in theorem 2.7 that $Z_{k}=\emptyset$ by computing the length of the scheme defined by the Jacobian ideal of $D_{k}$. More generally we can describe, at least when the base locus is smooth, the scheme $Z_{k}$ for any union of curves of the pencil.

Theorem 2.10. Assume that the base locus of the pencil $\mathcal{C}(f, g)$ is smooth and that the Jacobian ideal of $D^{s g}$ is locally a complete intersection. Assume also that $D_{k}$ contains all the singular members of the pencil except the singular curves $C_{\alpha_{i}, \beta_{i}}$ for $i=1, \ldots, r$. Then,

$$
\mathcal{J}_{Z_{k}}=\mathcal{J}_{\nabla C_{\alpha_{1}, \beta_{1}}} \otimes \cdots \otimes \mathcal{J}_{\nabla C_{\alpha_{r}, \beta_{r}}} .
$$

Remark 2.11. When $r=0$ we obtain the freeness again.

Proof. Since the set of singular points of two distinct curves are disjoint, it is enough to prove it for $r=1$. We recall that $\mathcal{E} x t^{1}\left(\mathcal{J}_{Z}, \mathscr{O}_{\mathbb{P}^{2}}\right)=\omega_{Z}$ where $Z$ is a finite scheme and $\omega_{Z}$ is its dualizing sheaf (see [2, Chapter III, section 7]); When the finite scheme $Z$ is locally a complete intersection, $\omega_{Z}=\mathscr{O}_{Z}$.

The dual exact sequence of

$$
0 \longrightarrow \mathcal{J}_{Z_{k}}(n(2-k)-1) \longrightarrow \mathcal{F} \longrightarrow \mathcal{J}_{\nabla D_{k}}(n k-1) \longrightarrow 0
$$

is the long exact sequence

$$
\begin{gathered}
0 \longrightarrow \mathscr{O}_{\mathbb{P}^{2}}(1-n k) \longrightarrow \mathscr{O}_{\mathbb{P}^{2}}(1-n)^{2} \longrightarrow \mathscr{O}_{\mathbb{P}^{2}}(n(k-2)+1) \longrightarrow \omega_{D_{k}} \longrightarrow \mathscr{O}_{\mathrm{sg}(\mathcal{F})} \longrightarrow \omega_{Z_{k}} \longrightarrow 0 \\
\longrightarrow \omega_{\nabla D} \longrightarrow
\end{gathered}
$$

Since $Z_{k}$ is the zero set of a rank two vector bundle it is locally a complete intersection and $\omega_{Z_{k}}=\mathscr{O}_{Z_{k}}$. The map $\mathcal{F} \longrightarrow \mathcal{J}_{\nabla D_{k}}(n k-1)$ can be described by composition; indeed it is given by two polynomials $(U, V)$ such that

$$
(U, V) \cdot(\nabla f, \nabla g)=\nabla\left(\prod_{i}\left(\alpha_{i} f+\beta_{i} g\right)\right) .
$$

We find, $U=\sum_{i} \alpha_{i} \prod_{j \neq i}\left(\alpha_{j} f+\beta_{j} g\right)$ and $V=\sum_{i} \beta_{i} \prod_{j \neq i}\left(\alpha_{j} f+\beta_{j} g\right)$. The complete intersection $T=\{U=0\} \cap\{V=0\}$ defines a multiple structure of length $n^{2}(k-1)^{2}$ along the base locus. It implies that the scheme defined by the Jacobian ideal $\mathcal{J}_{\nabla D_{k}}$ contains the scheme $T$. In other words $\omega_{\nabla D_{k}}=\mathscr{O}_{T} \oplus \mathfrak{R}$ where $\mathfrak{R}$ is supported by the singularities of $D_{k}$ outside the base locus of the pencil. Then we have

$$
0 \longrightarrow \mathfrak{R} \longrightarrow \mathscr{O}_{\mathrm{sg}(\mathcal{F})} \longrightarrow \mathscr{O}_{Z_{k}} \longrightarrow 0 \text {. }
$$

The hypothesis oon the nature of the singularities implies that $\mathfrak{R}=\oplus_{i=2, \ldots, s} \mathscr{O}_{Z_{\alpha_{i}, \beta_{i}}}$ and $\mathscr{O}_{\operatorname{sg}(\mathcal{F})}=\oplus_{i=1, \ldots, s} \mathscr{O}_{Z_{\alpha_{i}, \beta_{i}}}$. This proves $\omega_{Z_{k}}=\mathscr{O}_{Z_{\alpha_{1}, \beta_{1}}}$.

There are exact sequences relating the vector bundles $\mathcal{T}_{D_{k}}$ and $\mathcal{T}_{D_{k} \backslash C}$ when $C \subset D_{k}$.

Proposition 2.12. We assume that the base locus of the pencil $\mathcal{C}(f, g)$ is smooth, that the Jacobian ideal of $D^{s g}$ is locally a complete intersection and that $D_{k}$ contains $D^{\mathrm{sg}}$. Let $C$ be a singular member in $\mathcal{C}(f, g)$ and $Z$ its scheme of singular points. Then there is an exact sequence

$$
0 \longrightarrow \mathcal{T}_{D_{k}} \longrightarrow \mathcal{T}_{D_{k} \backslash C} \longrightarrow \mathcal{J}_{Z / C}(n(3-k)-1) \longrightarrow 0,
$$

where $\mathcal{J}_{Z / C} \subset \mathscr{O}_{C}$ defines $Z$ into $C$. 
Proof. The derivation $(\nabla f \wedge \nabla g)$. $\nabla$ is tangent to $D_{k}$ then also to $D_{k} \backslash C$. It induces the following commutative diagram which proves the proposition:

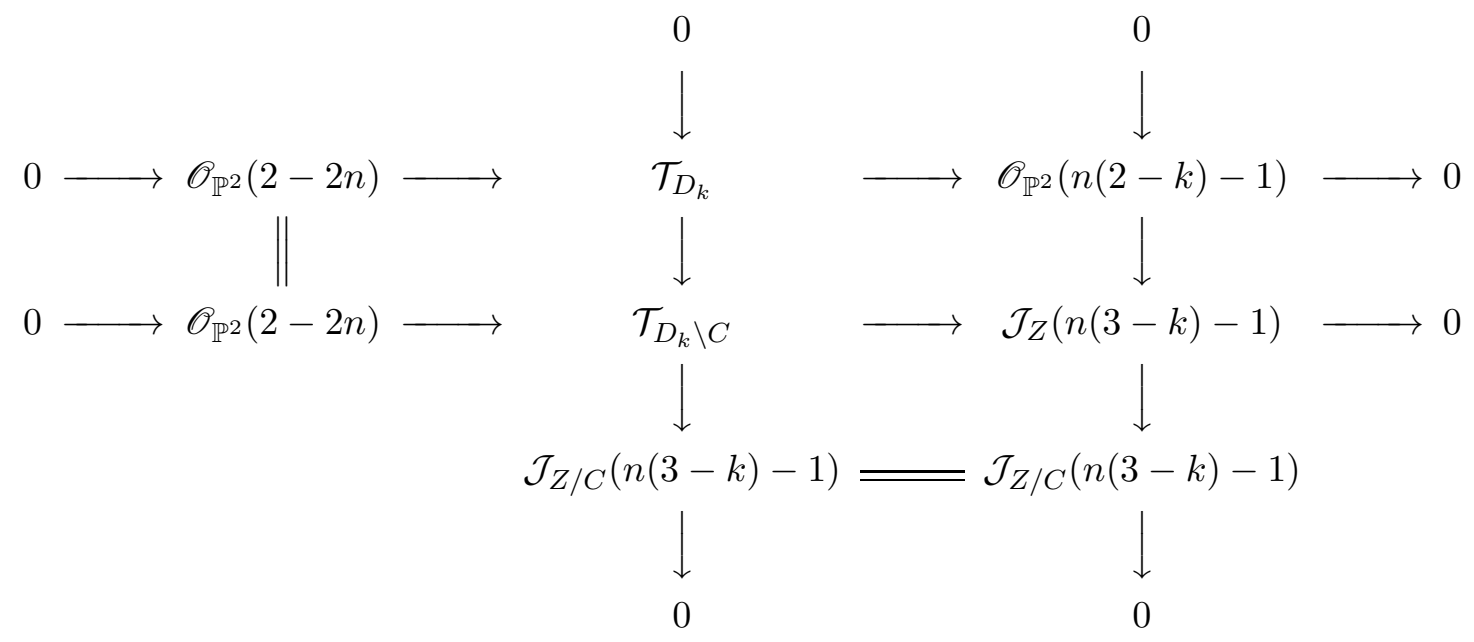

\section{The PENCIL CONTAins a NON-REDUCED CURVE}

When the pencil $\mathcal{C}(f, g)$ contains a non-reduced curve, the arguments used in the previous sections are not valid since the scheme defined by the Jacobian ideal contains a divisor. We have to remove this divisor somehow. Remember that if two curves of the pencil are multiple then the general curve is singular. So let us consider that there is only one curve that is not reduced. Let $h h_{1}^{r_{1}} \cdots h_{s}^{r_{s}}=0$ be the equation of this unique nonreduced curve where $h=0$ is reduced, $\operatorname{deg}\left(h_{i}\right)=m_{i} \geq 1$ and $r_{i} \geq 2$. Since the derivation $\frac{1}{\prod_{i} h_{i}^{r_{i}}}(\nabla f \wedge \nabla g) . \nabla$ is still tangent to all curves of the pencil, we believe that the following statement is true:

Conjecture. Let $h h_{1}^{r_{1}} \cdots h_{s}^{r_{s}}=0$ be the equation of the unique non-reduced curve where $\{h=0\}$ is reduced, $\operatorname{deg}\left(h_{i}\right)=m_{i} \geq 1$ and $r_{i} \geq 2$.

Then, $D_{k}$ is free with exponents $\left(2 n-2-\sum_{i=1}^{i=s}\left(r_{i}-1\right) m_{i}, n(k-2)+1\right)$ if and only if $D_{k} \supseteq D^{\mathrm{sg}}$ and the Jacobian ideal of the reduced structure of $D_{k}$ is locally a complete intersection.

\section{EXAMPLES}

Let us call $\Sigma_{3} \subset \mathbb{P}^{9}=\mathbb{P}\left(\mathrm{H}^{0}\left(\mathscr{O}_{\mathbb{P}^{2}}(3)\right)\right)$ the hypersurface of singular cubics. It is well known that its degree is 12 (see [3], for instance).

- Pappus arrangement freed by nodal cubics: Let us consider the divisor of the nine lines appearing in the Pappus arrangement; this divisor is the union of three triangles $T_{1}, T_{2}, T_{3}$ with nine base points. The pencil generated by $T_{1}$ and $T_{2}$ contains 3 triangles (each one represents a triple point in $\Sigma_{3}$ ); since $9<12$, singular cubics are missing in the pencil. There is no other triangle and no smoth conic+line in the Pappus pencil, when it is general enough. We can conclude that the missing cubics are, in general, nodal cubics $C_{1}, C_{2}, C_{3}$.

Let $D=T_{1} \cup T_{2} \cup T_{3} \cup C_{1} \cup C_{2} \cup C_{3}$ be the union of all singular fibers in the pencil generated by $T_{1}$ and $T_{2}$. Then, according to theorem 2.7 we have

$$
\mathcal{T}_{D}=\mathscr{O}_{\mathbb{P}^{2}}(-4) \oplus \mathscr{O}_{\mathbb{P}^{2}}(-13) \text {. }
$$


- Pappus arrangement: Let $T_{1} \cup T_{2} \cup T_{3}$ be the divisor consisting of the nine lines of the projective Pappus arrangement and $D=T_{1} \cup T_{2} \cup T_{3} \cup C_{1} \cup C_{2} \cup C_{3}$ be the union of all singular fibers in the pencil generated by two triangles among the $T_{i}$ 's. Let us call $K:=C_{1} \cup C_{2} \cup C_{3}$ the union of the nodal cubics and $z_{1}, z_{2}, z_{3}$ their nodes. Then we have, according to theorem 2.10, $\mathcal{T}_{D}=\mathscr{O}_{\mathbb{P}^{2}}(-4) \oplus \mathscr{O}_{\mathbb{P}^{2}}(-13)$ and an exact sequence

$$
0 \longrightarrow \mathscr{O}_{\mathbb{P}^{2}}(-4) \longrightarrow \mathcal{T}_{D \backslash K} \longrightarrow \mathcal{I}_{z_{1}, z_{2}, z_{3}}(-4) \longrightarrow 0 .
$$

The logarithmic bundle $\mathcal{T}_{D \backslash K}$ associated to the Pappus configuration is semi-stable and its divisor of jumping lines is the triangle $z_{1}^{\vee} \cup z_{2}^{\vee} \cup z_{3}^{\vee}$ as it is proved by retricting the above exact sequence to any line through one of the zeroes.

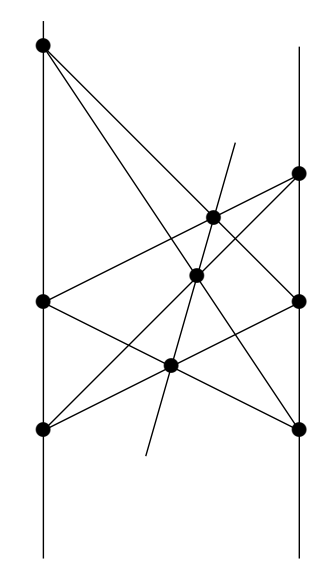

FiguRE 1. Pappus arrangement

- Hesse arrangement: Let us consider the pencil generated by a smooth cubic $C$ and its hessian Hess $(C)$. The pencil contains 4 triangles $T_{1}, T_{2}, T_{3}, T_{4}$ and since the degree of $\Sigma_{3}$ is 12 , no other singular cubic can be present. Let us call $D$ the union of these four triangles. Then, according to theorem 2.7 we have

$$
\mathcal{T}_{D}=\mathscr{O}_{\mathbb{P}^{2}}(-4) \oplus \mathscr{O}_{\mathbb{P}^{2}}(-7)
$$

\section{REFERENCES}

[1] Dimca, A. and Sernesi, E. Syzygies and logarithmic vector fields along plane curves, J. Éc. polytech. Math. 1 (2014), 247-267.

[2] Hartshorne, R. Algebraic geometry. Graduate Texts in Mathematics, 52. Springer-Verlag, 1977.

[3] Gelfand, I. M., Kapranov, M. M. and Zelevinsky, A. V. Discriminants, resultants, and multidimensional determinants. Mathematics: Theory and Applications. Birkhäuser Boston, Inc., Boston, MA, 1994.

[4] Okonek, C., Schneider, M. and Spindler, H. Vector bundles on complex projective spaces. Progress in Mathematics, 3. Birkhäuser, Boston, Mass., 1980.

[5] Orlik, P. and Terao, H. Arrangements of hyperplanes. Grundlehren der Mathematischen Wissenschaften, 300. Springer-Verlag, Berlin, 1992.

[6] Reiffen, H. Das Lemma von Poincaré für holomorphe Differentialformen auf komplexen Räumen, Math. Z., 101, (1967), 269-284.

[7] Saito, K. Theory of logarithmic differential forms and logarithmic vector fields, J. Fac. Sci. Univ. Tokyo, Sect. IA Math. 27 (1980), 265-291. 
[8] Schenck, H. A rank two vector bundle associated to a three arrangement and its Chern polynomial, Adv. Math., 149, (2000), no. 2, 214-229.

[9] Schenck, H. and Tohăneanu, Ş. O. Freeness of conic-line arrangements in $\mathbb{P}^{2}$. Comment. Math. Helv. 84 (2009), no. 2, 235-258.

[10] Simis, A. and Tohăneanu, Ş. O. Homology of homogeneous divisors. Israel J. Math. 200 (2014), no. 1, 449-487

[11] Terao, H. Arrangements of hyperplanes and their freeness. I.II. J. Fac. Sci. Univ. Tokyo Sect. IA Math. 27 (1980), no. 2, 293-312.

[12] Tevelev, E. A. Projective duality and homogeneous spaces. Encyclopaedia of Mathematical Sciences, 133, Invariant Theory and Algebraic Transformation Groups, IV, Springer-Verlag, Berlin, 2005.

E-mail address: jean.valles@univ-pau.fr

Université de Pau et des Pays de l'Adour, Avenue de l’Université - BP 576 - 64012 PAU Cedex - France

$U R L:$ http://jvalles.perso.univ-pau.fr/ 